\title{
Fetal operation followed by Caesarean section may have a beneficial effect upon neuromuscular function in spina bifida aperta Renate Verbeek*1, Axel Heep ${ }^{2}$, Natasha Maurits ${ }^{1}$, Reinhold Cremer ${ }^{3}$, Oebele Brouwer ${ }^{1}$, Johannes van der Hoeven ${ }^{1}$ and Deborah Sival ${ }^{4}$
}

\author{
Address: ${ }^{1}$ Department of Neurology, University Medical Center Groningen, University of Groningen, Hanzeplein 1, PO Box $30001,9700 R B$ \\ Groningen, the Netherlands, ${ }^{2}$ Department of Neonatology, University of Bonn, Sigmund Freud Str. 25, 53105, Bonn, Germany, ${ }^{3}$ Department of \\ Pediatrics, Children's Hospital Cologne, Amsterdamer Str. 59, 50735 Cologne, Germany and ${ }^{4}$ Department of Paediatrics, University Medical \\ Center Groningen, University of Groningen, Hanzeplein 1, PO Box 30001, 9700RB Groningen, the Netherlands \\ Email: Renate Verbeek* - r.j.verbeek@neuro.umcg.nl \\ * Corresponding author
}

from 53rd Annual Meeting of the Society for Research into Hydrocephalus and Spina Bifida Belfast, UK. 24-27 June 2009

Published: 27 November 2009

Cerebrospinal Fluid Research 2009, 6(Suppl 2):S38 doi:10.1186/1743-8454-6-S2-S38

This abstract is available from: http://www.cerebrospinalfluidresearch.com/content/6/S2/S38

(c) 2009 Verbeek et al; licensee BioMed Central Ltd.

\section{Background}

Spina bifida aperta (SBA) is associated with neurological dysfunction cranial and caudal to the meningomyelocele (MMC). Fetal surgery may ameliorate cranial abnormalities, but effects upon neuromuscular function caudal to the MMC are unclear. SBA myotomes cranial to the MMC are influenced by cerebral dysfunction, whereas myotomes caudal to the MMC are additionally influenced by the MMC. Increased muscle ultrasound density (MUD) reflects neuromuscular damage. By the intra-individual difference in MUD (dMUD = [MUDcaudal] - [MUDcranial]) the effect by the MMC upon neuromuscular integrity is derived. In the present study, we aimed to compare dMUD and neuromuscular function between fetally and postnatally-operated SBA patients.

\section{Materials and methods}

We compared dMUD and neuromuscular function in 6 (age- and MMC-) matched pairs of fetally and postnatally operated SBA patients [age 0-3.5 years; lumbar (6) and lumbar-sacral (6) MMC]. In all patients, quadriceps muscle was innervated cranial- and calf muscle caudal- to the MMC. Fetally operated patients were delivered by Caesarean section (CS) at Bonn; postnatally operated patients were delivered vaginally at Groningen.

\section{Results}

The dMUD was smaller in fetally than in postnatally-operated SBA patients [10 ( -11 to 37 ) vs 28 ( 4 to 47 ), medians (ranges); $p<0.05]$. Comparing SBA patients, the results indicated more preserved neuromuscular function in fetally than postnatally operated patients [median difference: 1 and 1.5 myotome (ranges 1-3); motor and sensory function resp.; $p<0.05)]$.

\section{Conclusion}

In SBA, dMUD provides a diagnostic tool to compare neuromuscular integrity between treatment groups. Present data suggest that fetal operation followed by CS has some beneficial effect upon neuromuscular outcome caudal to the MMC. Future assessment in SBA patients (treated by postnatal operation followed by CS) may allow further differentiation between the effect by operation and way of delivery upon neuromuscular outcome. 